\title{
Modeling Protein Structures with Conditional Random Fields: Final Report
}

\author{
Christopher James Langmead
}

Oct 28, 2008

\section{Personnel}

Approximately $75 \%$ of the funds were allocated to support one graduate student, Hetunandan Kamisetty, a Ph.D. student in the department of Computer Science. The research supported under this grant will form the bulk of his Ph.D. dissertation. The remainder of the funds were used to support travel to conferences, Summer salary support, and some basic hardware needs.

\section{Implementation}

The implementation of the software for modeling protein structures with fixed-backbones as graphical models is complete. We have implemented: (a) tools for converting PDB-style files into graphical models; (b) three different energy potentials; and (c) several algorithms for performing probabilistic inference and learning over graphical models. The software is available upon request, and it has been distributed to 7 research groups in 5 separate universities around the world.

\subsection{Energy Potentials}

In the past three years, we have implemented three force fields. The most sophisticated of these is nearly identical to the one used in the protein structure prediction program ROSETTA [14]. This has allowed us to perform head-to-head comparisons with ROSETTA on a number of tasks. In general, our method is more accurate than ROSETTA on key task, such as free energy calculations.

\subsection{Inference Algorithms}

We have implemented five algorithms for inference over graphical models: Belief Propagation (BP) [1, 10], Generalized Belief Propagation (GBP) [19], Naïve Mean Field [3], Tree-reweighted Belief Propagation [18], and a Gibbs sampler. While any of these algorithms can be used to perform probabilistic inference, each algorithm has its own special capabilities/features. Inference in BP and GBP, for example, have been shown to be a equivalent to the Bethe and Kikuchi approximations of the Gibbs free energy, respectively [19]. Mean-field and Tree-reweighted Belief Propagation, on the other hand, are capable of computing lower- and upper bounds on the partition function of the model, respectively.

\subsection{Learning Algorithm}

We have designed and implemented a novel learning algorithm for graphical models. This learning algorithm can re-estimate the parameters of a given force field by minimizing the difference between calculated and experimental free energies. Previous learning algorithms have considered minimizing differences in potential energies, which is a much easier problem. We have shown that re-estimating parameters according to free energies can improve accuracies substantially [5]. 


\section{Publications}

Over the three year project, we have published three papers $[8,6,9]$, and a fourth paper is presently under review. We have also published three technical reports [7, 11, 4]. Our first paper was published in the eleventh annual international conference on computational molecular biology (RECOMB) [8]. RECOMB is one of the top two conferences in computational biology with acceptance rates of $21 \%$. The paper demonstrated that a graphical-model approach to computing the free-energies of all-atom protein models is sufficiently accurate to perform non-trivial tasks (see Sec. 6). A technical report of that paper is also available [7]. A journal version of that paper was recently published as an invited paper in the Journal of Computational Biology [9]. A technical report extending the method to protein complexes was presented in [11]. We have also published a paper on new algorithms for computing rigorous upper and lower bounds on free energies using probabilistic inference [6]. Finally, we have submitted a paper to ISMB 2009 that extends our work to modeling protein complexes. The key results in that paper include: (i) the first graphical model for proteins with backbone flexibility, and (ii) the first learning algorithm for re-estimating the parameters of force fields by minimizing the differences in free energies. A technical report version of that submission [4].

\subsection{Additional Public Presentations}

The research supported by this grant has resulted in a total of 12 public presentations, including two presentations at international conferences by Mr. Hetunandan Kamisetty, and 10 invited talks by Dr. Langmead at various universities and conferences around the world.

\section{Patents}

No invention disclosures have been filed in relation to this work. We do, however, expect to file invention disclosures for the algorithms for designing proteins and drugs.

\section{Impact}

As a result of the publications and public presentations, a number of scientists have expressed interest in applying our approach to modeling biological macromolecules. Applications include: i) the identification of the transition-state ensemble in protein folding trajectories; ii) modeling nucleic acids; and iii) the design of protein sequences, and iv). Of these, items iii and iv are in the furthest state of development. The PI has established collaborations with Dr. Chris Bailey-Kellogg from the department of Computer Science at Dartmouth, and Dr. Ivet Bahar from the department of Computational Biology at the University of Pittsburgh. It is anticipated that these projects will last for multiple years and each will lead to multiple publications. Grant proposals surrounding this work have been submitted to various funding agencies including NSF, NIH, DOE, and Microsoft Research.

\section{Key Findings of Our Research}

In this section, we will briefly summarize some of the key findings we have made over the past year. 


\subsection{Fast Algorithms for Performing Free Energy Calculations}

Rigorous free energy calculations normally require days to weeks of computer time, often on super-computers. Our algorithms $[5,6,8,9]$, in contrast, are rigorous, but require only a few minutes of computer time, on a single-processor work station. The key distinction between our approach and those based on Molecular Dynamics simulations is that our algorithms do not require sampling or integration. Rather, it can can be shown that free energy approximations can be obtained by solving a particular optimization problem. That optimization problem, in turn, can be solved efficiently using inference algorithms for probabilistic graphical models.

The cost of traditional free energy calculations precludes so-called "high-throughput" applications, such as database screens. Our approach, in contrast, is orders of magnitude faster than traditional methods. Moreover, our algorithm can easily be parallelized, whereas Molecular Dynamics simulations cannot. We believe that an interesting area for future research is the creation of parallel codes for performing free energy calculations via probabilistic inference.

\subsubsection{Accuracy: Decoy Detection}

In the protein structure prediction literature, the term decoy is used to describe a protein structure that is plausible (i.e., one with low internal energy), but significantly different than the protein's true native structure. Decoys arise in practice when a given protein structure prediction program produces multiple predictions for the same sequence. Here, each prediction corresponds to a different local minimum in the protein's energy landscape. It is an open problem to devise an energy/scoring function that can reliably differentiate native structures from decoys. Indeed, it has been conjectured $[2,16]$ that energy functions comprising sums of pairwise interactions cannot distinguish a protein's native structure from decoy structures within about 1 $\AA$ RMSD. This is problematic because virtually all energy functions in use fall into this category owing to the fact that the physics are always described in terms of pairs of interacting particles.

In $[8,9]$ we hypothesized that the reason why it is difficult to identify a native structure amongst a set of decoys is that most techniques rely solely on the internal energy, $E$, associated with each structure. Physically, however, it makes more sense to consider the structure's free energy: $G=E-T S$, which is a combination of the internal energy and the product of the temperature, $T$, and the configurational entropy, $S$. Under the hypothesis, given a set of putative structures for the same protein with the same (or similar) internal energies, the native structure will be the one that has the largest entropy. Unfortunately, traditional methods for estimating the entropy of a given structure, including Molecular Dynamics or Monte-Carlo simulations, are costly. Free-energy calculations via GBP, on the other hand, are fast, running in a few minutes.

We tested our hypothesis and demonstrated that the native structure is indeed usually the one with lowest free energy. In particular, on a set of 48 different proteins, each having 35 decoys on average, the native fold is ranked \#1 $87.5 \%$ of the time. Moreover, the native structure was ranked in the top 4 of all structures $100 \%$ of the time (Fig. 1-A). A similar result was shown in [12] where protein-protein complexes were modeled, and the same free energy based approach was able to identify the correct binding mode; there, an accuracy of $80 \%$ was obtained across 5 protein-protein complexes, each having 11 decoys, on average. We believe these results support our hypothesis that it is important to consider entropic factors. Additionally, free-energy calculations using GBP take on the order of 5 minutes per structure, which compares very favorably to the hours (or even days) required by Molecular Dynamics or Monte Carlo-style free-energy calculations. Finally, in a direct comparison with 5 other methods for identifying native structures, our method outperforms the others (Fig. 1-B). 


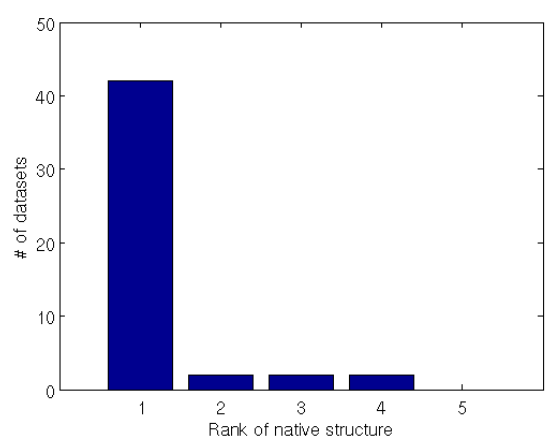

(a)

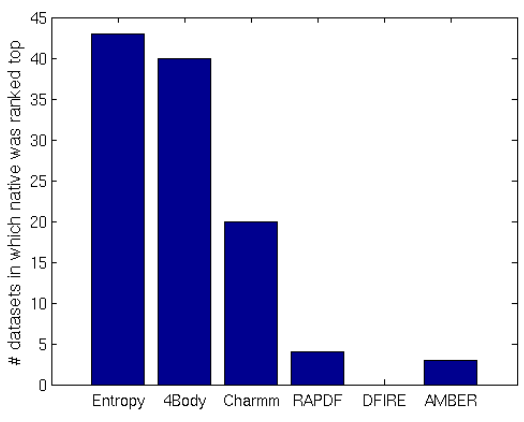

(b)

Figure 1: Decoy Detection (a) The histogram shows the distribution of the rank of the native structure, when ranked in decreasing order of the GBP-based entropy estimate, from among a set of 35 decoys (on average) for each of 48 immunoglobulin structures. Over this dataset, the native structure has the highest entropy $87.5 \%$ of the time. (b) Comparison of our method for identifying native structures versus 5 additional methods as reported in [15]. Our approach correctly identifies more native structures than other methods on a common set of proteins.

Our results present a counter-example to the conjecture $[2,16]$ that pairwise energy functions cannot identify native structures. We suggest that pairwise energy functions may be sufficient if a suitable entropic correction is computed.

\subsubsection{Accuracy: Correlation and Mean Squared Errors}

An additional test of the accuracy of the GBP-based energy calculations was conducted in the context of predicting the change in the change in free energy (i.e., $\Delta \Delta \mathrm{G}$ ) upon mutations. In $[8,9]$, we made predictions based on in-silico mutations and contrasted them with experimentally determined values as obtained from the PROTHERM [13] database of thermodynamic data. As seen in Figure 2, our predictions are wellcorrelated with experiment, achieving correlations between 0.63 and 0.7 .

More recently, the PI used graphical models to predict binding free-energies between proteins and ligands (unpublished results). The correlation coefficient between the predicted and experimental binding free energies was 0.714 , with a standard error of $1.7 \mathrm{kcal} / \mathrm{mol}$. Significantly, our method outperformed a state-of-the-art method (GOLD [17]) for estimating entropic contributions to the binding free energy.

Finally, we have recently extended our method to model protein complexes. In particular, we are looking at the problem of predicting the binding free energy. In a recent submission [5], we show that our free energy calculations are more than $1 \mathrm{kcal} /$ mole more accurate than ROSETTA, a state-of-the-art method for modeling proteins and protein complexes. This is a very large increase in accuracy. The two key advances in that paper are a new technique for modeling backbone flexibility, and a new algorithm for estimating force field parameters. 


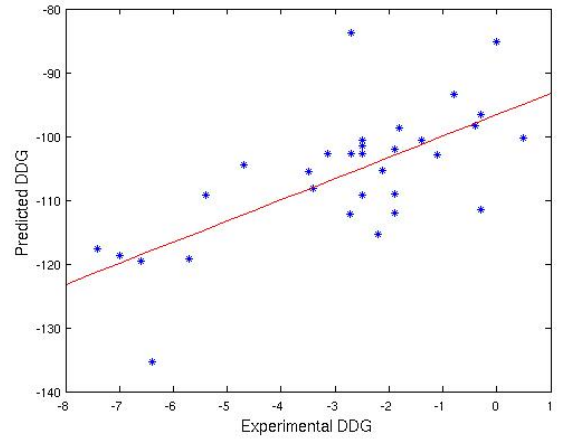

(a)

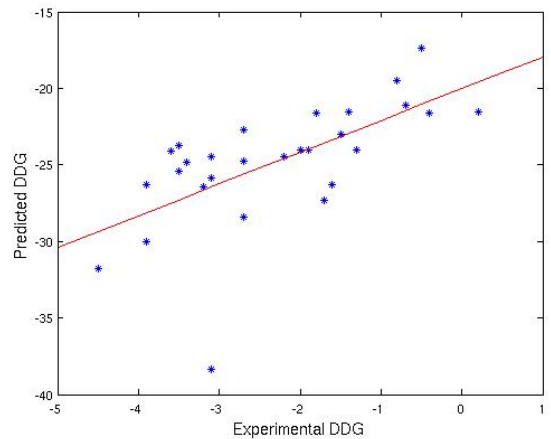

(b)

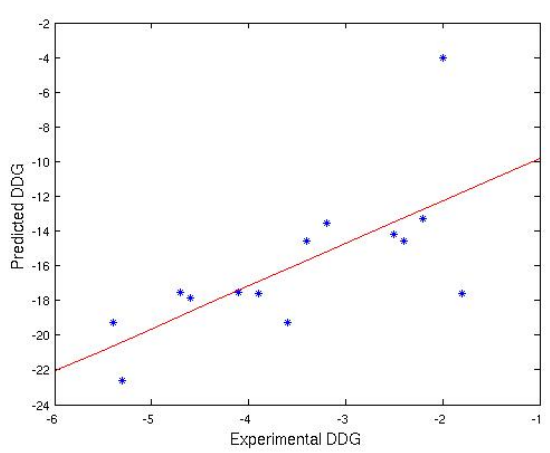

(c)

Figure 2: Plots showing variation of experimental $\Delta \Delta G$ (on the X-axis) with computed estimates of $\Delta \Delta G$, along with a least squares fit for (a) thirty one mutants of barnase (pdb id: $1 B N I$ ), $R=0.70, p=1.5^{*} 10^{-5}$ (b) twenty eight mutants of T4 Lysozyme(pdb id:1L63), $\mathrm{R}=0.63, \mathrm{p}=3.0^{*} 10^{-4}$ and (c) fourteen mutants of staphylococcal nuclease (pdb id:1STN), $R=0.69, p=0.0063$ 


\subsection{Upper and Lower Bounds on Partition Functions}

The GBP inference algorithm computes an approximation to the Gibbs free energy by computing an approximation to the partition function of the model. GBP does not, however, provide a guarantee to the quality of the estimate of the partition function. The Naïve Mean Field [3] and Tree-reweighted Belief Propagation [18] inference algorithms, on the other hand, compute lower and upper bounds on the partition function, respectively. Thus, we can evaluate the relative quality of GBP-based predictions by considering the ratio of the GBP estimates with these upper and lower bounds. In [6] performed that experiment on several proteins. We find that while the quality of the estimate does vary by protein, the upper-bound:GBP and GBP:lowerbound ratios never exceed 1.2. That is, our predictions are within $20 \%$ of the true value. This suggests that the GBP estimates are, in fact, near their true values and, somewhat surprisingly, that the upper and lower bounds are fairly close to each other.

\subsection{Generating Configurations}

We have implemented an algorithm which can generate the top $k$ most-likely structures, in order, for arbitrary $k$. Initially, we thought this would be a useful way to generate a diverse ensemble of structures near the lowest-energy configuration. Such ensembles have been shown to be useful in a number of different contexts, such as docking studies in computer-aided drug design. What we found, however, is that the difference in structure between the $k$ th-most likely structure, and the $(k+1)$ st-most likely structure is often simply a change in the configuration of a single side chain. That is, the method does not produce a diverse set of structures. We subsequently implemented a Gibbs sampler to generate diverse ensembles. However, the ability to generate structures in order of decreasing probability is still interesting and may one day find use in different domains.

\section{Ongoing Work and Future Directions}

Our work has attracted the attention of a variety of researchers. As previously mentioned, we have established collaborations with Drs. Chris Bailey-Kellogg and Dr. Ivet Bahar. Additionally, the PI has received a small grant from Microsoft Research to create a prototype model for modeling the emergence of resistance in HIV-1 protease. This project will develop algorithms for simulating the evolution of HIV-1 under the evolutionary pressure imposed by a drug. This work will adapt the algorithms for protein design. We are also exploring the possibility of designing allosteric proteins using our method. Specifically, we believe that the message-passing inference algorithms developed as part of this research can provide insights into how energy (and thus information) propagates throughout proteins. We are also interested in extending our model to include explicit solvent molecules, as opposed to the implicit solvation terms we presently use. Finally, we have recently devised a method for modeling protein dynamics using graphical models. This research will lead to efficient new ways of simulating the behaviors of molecules.

\section{References}

[1] AJi, S.M. AND MCELIECE, R.J. The Generalized Distributive Law. IEEE Transactions on Information Theory 46 (2000), 325-343. 
[2] Betancourt, M. R., And Thirumalai, D. Pair potentials for protein folding: choice of reference states and sensitivity of predicted native states to variations in the interaction schemes. Protein Science 8 (1999), 361-369.

[3] Jordan, M. I., Ghahramani, Z., JaAkkola, T., And Saul, L. K. An introduction to variational methods for graphical models. Machine Learning 37, 2 (1999), 183-233.

[4] Kamisetty, H., Bailey-Kellogg, C., And Langmead, C. A graphical model approach for predicting free energies of association for protein-protein interactions under backbone and side-chain flexibility. Tech. Rep. CMU-CS-08-162, Carnegie Mellon University School of Computer Science, 2008.

[5] Kamisetty, H., Bailey-Kellogg, C., And Langmead, C. A graphical model approach to predicting free energies of association for protein-protein interactions under backbone and side-chain flexibility. Submitted, RECOMB 2009 (2009).

[6] Kamisetty, H., And Langmead, C. Conformational Free Energy of Protein Structures: Computing Upper and Lower bounds. Proc. of 3DSIG 2008 Struct. Bioinf. and Computat. Biophysics, Toronto, $O N$. (2008), in press.

[7] Kamisetty, H., Xing, E., And Langmead, C. Free Energy Estimates of All-atom Protein Structures Using Generalized Belief Propagation. Tech. Rep. CMU-CS-06-160, Carnegie Mellon University, 2006.

[8] Kamisetty, H., Xing, E., And Langmead, C. Free Energy Estimates of All-atom Protein Structures Using Generalized Belief Propagation. In Proc. of the 7th Ann. Intl. Conf. on Research in Comput. Biol. (RECOMB) (2007), pp. 366-380.

[9] Kamisetty, H., Xing, E., And Langmead, C. Free Energy Estimates of All-atom Protein Structures Using Generalized Belief Propagation. J. Comp. Biol. 15, 7 (2008), 755-766.

[10] Kschichlang, F. R. And Frey, B.J. and Loeliger, H.A. Factor Graphs and the Sum-Product Algorithm. IEEE Transactions on Information Theory 47 (2001), 498-519.

[11] Langmead, C., And Kamisetty, H. Detecting protein-protein interaction decoys using fast free energy calculations. Tech. Rep. CMU-CS-07-156, Carnegie Mellon University, 2007.

[12] Langmead, C., And Kamisetty, H. Detecting Protein-Protein Interaction Decoys using Fast Free Energy Calculations. Tech. Rep. CMU-CS-07-156, Carnegie Mellon University, 2007.

[13] MD, K., KA, B., MM, G., P, P., K, K., H, U., AND A, S. ProTherm and ProNIT: thermodynamic databases for proteins and protein-nucleic acid interactions. Nuleic Acids Res. 34:D204-6, Database issue (2006).

[14] Rohl, C., Strauss, C., Misura, K., And Baker, D. Protein structure prediction using Rosetta. Methods Enzymol 383 (2004), 66-93.

[15] Summa, C., Levitt, M., And Degrado, W. An atomic environment potential for use in protein structure prediction. Journal of Mol. Bio. 352 (2005), 986-1001. 
[16] Tobi, D. Elber, R. Distance-dependent, pair potential for protein folding: Results from linear optimization. Proteins: Structure, Function and Genetics 41 (2000), 40-46.

[17] Verdonk, M. L., Cole, J. C., Hartshorn, M. J., Murray, C. W., and Taylor, R. D. Improved protein-ligand docking using gold. Proteins 52, 4 (2003), 609-623.

[18] Wainwright, M., JaAkkola, T., And Willsky, A. A new class of upper bounds on the log partition function, 2002.

[19] Yedidia, J., Freeman, W., AND Y., W. Constructing free-energy approximations and generalized belief propagation algorithms. IEEE Transactions on Information Theory 51 (2005), 2282-2312. 